\title{
Disponibilidade de acadêmicos de enfermagem para educação interprofissional em saúde
}

\author{
Nursing students' readiness for interprofessional health education
}

Disponibilidad de estudiantes de enfermería para la educación interprofesional en salud

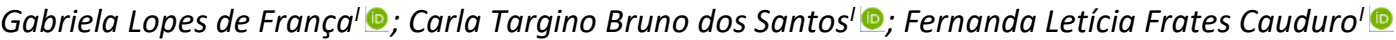

'Universidade de Brasília. Brasília, DF, Brasil

\begin{abstract}
RESUMO
Objetivo: avaliar a disponibilidade de acadêmicos de enfermagem para a educação interprofissional em saúde. Método: estudo realizado com acadêmicos de enfermagem de uma instituição pública de ensino superior do Distrito Federal. Os dados foram coletados no período de março a maio de 2020. Utilizou-se a escala Readiness for Interprofessional Learning Scale, versão adaptada para a língua portuguesa, composta por três fatores de avaliação. Adicionou-se uma questão aberta ao instrumento. Utilizaram-se duas técnicas de análise. Estatística, operacionalizada pelo software PSPP, e categorial. Protocolo de pesquisa aprovado pelo Comitê de ética em Pesquisa. Resultados: os 31 acadêmicos de enfermagem participantes apresentaram disponibilidade satisfatória para a educação interprofissional em saúde, observada nos três fatores avaliados. Obteve-se respostas positivas, com destaque para o item $3(87,1 \%)$ do fator 1 , e o item 29 (90, 3\%) do fator 3. Conclusão: a disponibilidade para a educação interprofissional em saúde foi avaliada como satisfatória.

Descritores: Enfermagem; Educação em Enfermagem; Educação Interprofissional; Práticas Interdisciplinares; Estudantes de enfermagem.
\end{abstract}

\begin{abstract}
Objective: to assess nursing students' readiness for interprofessional health education. Method: in this study with nursing students from a public higher education institution in the Federal District, data were collected from March to May 2020, using a version of the Readiness for Interprofessional Learning Scale adapted for Portuguese and consisting of 3 assessment factors. An open question was added to the instrument. The data were analyzed statistically, using PSPP software, and categorically. The research protocol was approved by the research ethics committee. Results: by the three factors evaluated, the 31 nursing students displayed satisfactory readiness for interprofessional health education. Positive responses were obtained, particularly on factor 1, item 3 (87.1\%), and factor 3, item 29 (90.3\%). Conclusion: readiness for interprofessional health education was found to be satisfactory.

Descriptors: Nursing; Education, Nursing; Interprofessional Education; Interdisciplinary Placement; Students, Nursing.
\end{abstract}

\section{RESUMEN}

Objetivo: evaluar la disponibilidad de estudiantes de enfermería para la educación interprofesional en salud. Método: estudio realizado con estudiantes de enfermería de una institución pública de educación superior del Distrito Federal. Los datos fueron recolectados de marzo a mayo de 2020. Se utilizó la Readiness for Interprofessional Learning Scale, una versión adaptada para el idioma portugués, que cuenta con 3 factores de evaluación. Se agregó una pregunta abierta al instrumento. Se utilizaron dos técnicas de análisis. Estadística, operadas por software PSPP, y categórica. Protocolo de investigación aprobado por el Comité de Ética en Investigación. Resultados: los 31 estudiantes de enfermería que participaron presentaron disponibilidad satisfactoria en cuanto a la educación interprofesional en salud, observada en los tres factores evaluados. Se obtuvieron respuestas positivas, con énfasis al ítem $3(87,1 \%)$ del factor 1 , y el ítem 29 (90,3\%) del factor 3 . Conclusión: se evaluó la disponibilidad para la educación interprofesional en salud como siendo satisfactoria.

Descriptores: Enfermería; Educación en Enfermería; Educación Interprofesional; Prácticas Interdisciplinarias; Estudiantes de Enfermería.

\section{INTRODUÇÃO}

A interprofissionalidade ocorre quando profissionais de diferentes áreas da saúde aprendem conjuntamente entre $\mathrm{si}^{1}$. Ela promove melhores condições no trabalho, na qualidade da assistência e contribui para as práticas de equipe ${ }^{2}$. As constantes mudanças no cenário da atenção à saúde no Brasil têm propiciado amplos debates sobre a formação profissional e impulsionado instituições de ensino superior a revisitar e discutir seus modelos pedagógicos. Desde a constituição do Sistema Único de Saúde (SUS), a atuação em equipes e redes tem sido colocada como uma estratégia para fortalecimento do trabalho em saúde. Nesse sentido, conceitos como multidisciplinaridade, interdisciplinaridade, transdisciplinaridade e interprofissionalidade passaram a ganhar espaço, seja no campo acadêmico ou profissional ${ }^{3}$.

Agradecimento ao Programa Institucional de Bolsas de Iniciação Científica, Tecnológica e de Inovação (PIBIC) da Fundação de Apoio à Pesquisa do Distrito Federal (FAPDF), protocolo número 25418.96.43239.12092018-5154.

Autora correspontente: Gabriela Lopes de França. E-mail: gabriela.loppesss@gmail.com

Editora Científica: Cristiane Helena Gallasch; Editora Associada: Helena Maria Scherlowski Leal David 
No contexto da formação de enfermeiros, o impacto pode ser visualizado nas Diretrizes Curriculares Nacionais (DCN) de 2001, a qual estabelece os eixos norteadores para a formação profissional e destaca a importância do trabalho multi e interdisciplinar ${ }^{4}$. Em 2017, o Conselho Nacional de Saúde, ao apresentar os princípios a serem incorporados nas novas DCN dos cursos de graduação da área da saúde, explicita a educação e o trabalho interprofissional como meio para superação dos desafios do trabalho em saúde ${ }^{5}$.

A educação interprofissional (EIP) é compreendida como o processo de construção do conhecimento entre estudantes de duas ou mais profissões que aprendem com, sobre e entre si, a fim de possibilitar a colaboração e melhorar os resultados no âmbito da saúde. Entidades como a Organização Mundial da Saúde (OMS) tem se esforçado na disseminação de informações e programas para o estímulo e adoção de iniciativas em EIP. Isto pois, quando implementada na graduação, há possibilidade dos estudantes desenvolverem habilidades de comunicação, análise crítica, valorização dos desafios e reconhecimento das vantagens do trabalho em equipe . $^{6}$

No Brasil, os estudos ${ }^{2,7,8}$ tem favorecido a divulgação da temática e possibilitado a inserção da EIP na agenda acadêmica com fomento ao desenvolvimento de pesquisas. Embora essas publicações exponham a necessidade da incorporação da EIP nos cenários de formação em saúde, ainda são incipientes universidades, escolas e programas que tenham sua abordagem pautadas nos princípios do interprofissionalismo ${ }^{9}$. Um estudo divulgou que no Brasil, dos 883 cursos de enfermagem e 197 cursos de medicina, apenas uma universidade pública inseriu em seu currículo a EIP, e um pequeno número de graduações implementam seus princípios em ações desenvolvidas para a formação dos alunos ${ }^{9}$.

Para além da incorporação da EIP pelas instituições de ensino, também se faz necessário averiguar as atitudes e expectativas dos estudantes para o trabalho interprofissional. Nesse sentido, a Readiness for Interprofessional Learning Scale (RIPLS) é uma escala que possibilita investigar a disponibilidade de acadêmicos da área da saúde para o conhecimento compartilhado ${ }^{9}$.

A âmbito nacional, estudos têm apresentado resultados com a aplicação da RIPLS ${ }^{10-17}$, dos quais quatro investigaram acadêmicos de enfermagem ${ }^{12,13,15,17}$. Face ao exposto, o objetivo dessa pesquisa foi avaliar a disponibilidade de acadêmicos de enfermagem para a educação interprofissional em saúde.

\section{MÉTODO}

Estudo quantitativo, descritivo e exploratório, realizado em uma Universidade Pública do Centro-Oeste do Brasil. A população alvo foi composta pelos 42 acadêmicos de enfermagem regularmente matriculados no oitavo semestre do curso de graduação. Optou-se por essa estratificação, pois esta etapa do curso antecede o estágio curricular obrigatório, momento em que os acadêmicos têm ampla oportunidade de atuar com estudantes e profissionais de outras áreas da saúde.

Foram inclusos aqueles que aceitaram participar formalmente da pesquisa por meio do preenchimento do Termo de Consentimento Livre e Esclarecido (TCLE). Como critérios de exclusão, acadêmicos não matriculados regularmente no curso e que em algum momento expressaram desejo em não mais participar da pesquisa. A abordagem dos potenciais participantes ocorreu de forma virtual, através de e-mail e aplicativo de mensagem. A amostra final foi composta por 31 acadêmicos.

A coleta de dados foi realizada entre março e maio de 2020, com a aplicação da escala RIPLS, versão traduzida e validada para o contexto brasileiro ${ }^{9}$, ajustada para o Google Forms ${ }^{\circledR}$. Para a caracterização dos participantes foram incluídas questões de dados sociodemográficos. A fim de enriquecer a produção de dados, a questão aberta "O que é interprofissionalidade?" foi acrescentada ao instrumento.

Foi utilizada a versão da RIPLS em português composta por 32 itens, sendo quatro itens reversos ${ }^{18}$, agrupados em três fatores: Fator 1-Trabalho em equipe e colaboração; Fator 2 - Identidade Profissional e Fator 3 - Atenção à saúde centrada no paciente. Cada questão é avaliada pelo respondente por meio de escala de Likert cujos número/ rótulos semânticos são dispostos em "Discordo totalmente (1)", "Discordo (2)", "Nem concordo, nem discordo" (3), "Concordo (4)" e "Concordo plenamente (5)". Quanto maior o escore, maior é a disponibilidade para a aprendizagem interprofissional ${ }^{5}$. O item 12 (fator 1 ) e os itens 10, 11 e 21 (fator 2 ) remetem a atitudes negativas perante à EIP, sendo analisados de forma reversa.

Os dados obtidos foram organizados em planilha do Excel e a análise estatística descritiva dos dados numéricos, média e desvio-padrão, realizada por meio do software PSPP versão 1.2.0-g0fb4db. Para interpretação das médias, utilizou-se a classificação, zona de perigo $(1,00$ a 2,33$)$; zona de alerta $(2,34-3,66)$ e zona de conforto $(3,67-5,00)$. Se as médias se encontram na zona de conforto recomenda-se que haja manutenção das atitudes. Caso estejam na zona de alerta deve haver aprimoramento, e na zona de perigo são necessárias mudanças urgentes ${ }^{19}$.

Para as respostas à questão aberta optou-se por análise categorial das unidades de registro, a qual ocorreu em três etapas: pré-análise; exploração do material ou codificação e tratamento dos resultados - inferência e interpretação ${ }^{20}$. Para 
a inferência e interpretação adotaram-se estudos e documentos de referência em pesquisa na temática de EIP e interprofissionalidade ${ }^{1,21,22}$. Adotou-se a letra $E$ para denominação dos acadêmicos participantes da pesquisa.

O protocolo de pesquisa teve aprovação do Comitê de Ética em Pesquisa da instituição, seguindo a Resolução no 466/12 do Conselho Nacional de Saúde ${ }^{23}$.

\section{RESULTADOS}

Participaram do estudo 31 estudantes, sendo a maioria do sexo feminino ( $n=26 ; f=83,9 \%$ ), com idade entre 21 e 36 anos, e ingresso na universidade no ano de 2016 ( $n=21 ; f=67,7 \%$ ).

Na Tabela 1 são apresentados, por fatores, os resultados obtidos a partir da aplicação da escala RIPLS. A Tabela 2 demonstra as médias de 4,63 para o fator 1, 4,32 para o fator 2, e 4,69 para o fator 3, correspondendo a classificação de zona de conforto.

TABELA 1: Distribuição das respostas dos acadêmicos do curso de enfermagem nos itens da Readiness for Interprofessional Learning Scale. Brasília, DF, Brasil, 2020.

\begin{tabular}{|c|c|c|c|c|}
\hline Fatores/Itens RIPLS & & ont & $\begin{array}{l}\text { de } \\
\text { Esc }\end{array}$ & $\begin{array}{l}\text { ost } \\
\text { ker }\end{array}$ \\
\hline & 1 & 2 & 3 & 4 \\
\hline
\end{tabular}

\section{Fator 1. Trabalho em equipe e colaboração}

1 A aprendizagem junto com outros estudantes ajudará a me tornar um participante mais efetivo de uma equipe de saúde.

$2 \mathrm{Em}$ última análise os pacientes seriam beneficiados se estudantes da área da saúde trabalhassem juntos para resolver os problemas dos pacientes.

3 Aprendizagem compartilhada com outros estudantes da área da saúde aumentará minha capacidade de compreender problemas clínicos.

$4 \mathrm{~A}$ aprendizagem junto com outros estudantes da área da saúde durante a graduação melhoraria os relacionamentos após a graduação.

5 Habilidades de comunicação deveriam ser aprendidas junto com outros estudantes da área da saúde.

6 A aprendizagem compartilhada me ajudará a pensar positivamente sobre outros profissionais.

7 Para que a aprendizagem em pequenos grupos funcione, os estudantes precisam confiar e respeitar uns aos outros.

8 Habilidades de trabalho em equipe são essenciais na aprendizagem de todos os estudantes da área da saúde.

9 A aprendizagem compartilhada me ajudará a compreender minhas próprias limitações.

12 Habilidades para solução de problemas clínicos só devem ser aprendidas com estudantes do meu próprio curso.

13 A aprendizagem compartilhada com estudantes de outras profissões da saúde ajudará a me comunicar melhor com os pacientes e outros profissionais.

14 Gostaria de ter a oportunidade de trabalhar em projetos, em pequenos grupos, com estudantes de outras profissões da saúde

15 A aprendizagem compartilhada ajudará a esclarecer a natureza dos problemas dos pacientes.

16 A aprendizagem compartilhada durante a graduação me ajudará a tornar-me um profissional que trabalha melhor em equipe

40 É importante que os profissionais da saúde estabeleçam objetivos comuns para o trabalho em equipe.

\section{Fator 2. Identidade profissional}

10 Evito desperdiçar meu tempo aprendendo junto com estudantes de outras profissões da saúde.

11 É desnecessário que estudantes de graduação da área da saúde aprendam juntos.

\begin{tabular}{|c|c|c|c|c|}
\hline ---- & ---- & 6,4 & 12,9 & 80,6 \\
\hline 3,2 & 3,2 & 3,2 & 9,6 & 80,6 \\
\hline ---- & ---- & 3,2 & 9,6 & 87,1 \\
\hline 3,2 & ---- & 6,4 & 6,4 & 83,8 \\
\hline ---- & ---- & 3,2 & 32,2 & 64,5 \\
\hline ---- & ---- & 9,6 & 32,2 & 58,0 \\
\hline ---- & ---- & 9,6 & 16,1 & 74,1 \\
\hline ----- & ---- & 3,2 & 16,1 & 80,6 \\
\hline ---- & ---- & 3,2 & 32,2 & 64,5 \\
\hline 45,1 & 29,0 & 12,9 & ---- & 12,9 \\
\hline ---- & ---- & ---- & 25,8 & 74,1 \\
\hline ---- & ---- & ---- & 22,5 & 77,4 \\
\hline ---- & 3,2 & 3,2 & 22,5 & 70,9 \\
\hline ---- & ---- & ---- & 19,3 & 80,6 \\
\hline ---- & ---- & 6,4 & 25,8 & 67,7 \\
\hline 64,5 & 29,0 & 3,2 & ---- & 3,2 \\
\hline 70,9 & 16,1 & ---- & 3,2 & 9,6 \\
\hline 54,8 & 19,3 & 12,9 & 9,6 & 3,2 \\
\hline ---- & ---- & 16,1 & 38,7 & 45,1 \\
\hline
\end{tabular}

21 Eu me sentiria desconfortável se outro estudante da área da saúde soubesse mais sobre um tópico que eu.

38 Para desenvolver minhas atividades profissionais, é importante conhecer as atribuições dos outros profissionais da saúde.

Fonte: Dados da Pesquisa, 2020. 
TABELA 1: Distribuição das respostas dos acadêmicos do curso de enfermagem nos itens da Readiness for Interprofessional Learning Scale. Brasília, DF, Brasil, 2020 (continuação)

\section{Fatores/Itens RIPLS}

Porcentagem de resposta po

Fator 3. Atenção à saúde centrada no paciente

25 Gosto de entender o problema na perspectiva do paciente (situação do paciente).

1

$2 \quad 3 \quad 4 \quad 5$

26 Estabelecer uma relação de confiança com meu paciente é importante para mim (situação do paciente).

27 Procuro transmitir compaixão aos meus pacientes (situação do paciente).

28 Pensar no paciente como uma pessoa é importante para

indicar o tratamento correto (situação do paciente).

29 Na minha profissão são necessárias habilidades de interação e cooperação com os pacientes (situação do paciente).

310 paciente é corresponsável pelo cuidado.

32 A qualidade do cuidado prestado ao Paciente depende de conhecimentos e habilidades de diversas profissões da saúde.

33 A opinião do paciente pode mudar minha conduta clínica.

34 A articulação entre os profissionais de saúde é fundamental para a qualidade do cuidado ao paciente.

35 Compreender o contexto de vida do paciente contribui para a qualidade do cuidado.

36 A família do paciente deve participar do cuidado.

370 vínculo do profissional com o paciente e sua família influencia a qualidade do cuidado.

$-\quad--\quad 9,6 \quad 29,0 \quad 61,2$

39 O paciente deve participar das decisões sobre seu

plano terapêutico

\begin{tabular}{|c|c|c|c|c|}
\hline ---- & ----- & 3,2 & 9,6 & 87,1 \\
\hline ---- & 3,2 & 16,1 & 12,9 & 67,7 \\
\hline ---- & ---- & ---- & 19,3 & 80,6 \\
\hline & ---- & ---- & 9,6 & 90,3 \\
\hline & 3,2 & 6,4 & 12,9 & 77,4 \\
\hline & 3,2 & 3,2 & 6,4 & 87,1 \\
\hline - & 3,2 & 16,1 & 32,2 & 48,3 \\
\hline & ---- & ---- & 16,1 & 83,8 \\
\hline & --- & --- & 16,1 & 83,8 \\
\hline & --- & 3,2 & 16,1 & 80,6 \\
\hline & ---- & 9,6 & 19,3 & \\
\hline
\end{tabular}

Fonte: Dados da Pesquisa, 2020.

Embora sejam evidenciadas respostas positivas para a maioria dos itens que compõem a escala, no fator 1 "Trabalho em equipe e colaboração", destacam-se os itens 3 "Aprendizagem compartilhada com outros estudantes da área da saúde aumentará minha capacidade de compreender problemas clínicos", o qual obteve concordância total para $87,1 \%$ dos respondentes, seguido pelos itens $4(83,8 \%), 1(80,6 \%), 2(80,6 \%), 8(80,6 \%)$ e 16 (80,6\%) os quais enfatizam a pertinência de serem oportunizados, durante a formação acadêmica, espaços de aprendizagem colaborativa e compartilhada para o desenvolvimento de habilidades essenciais ao trabalho em equipe e seus benefícios ao cuidado dos pacientes. Maior variabilidade nas respostas foi identificada no item reverso 12 , "Habilidades para solução de problemas clínicos só deve ser aprendida com estudantes do meu próprio curso".

O fator 2, "Identidade profissional" concentra quatro itens, destes, três reversos, dos quais o item "Eu me sentiria desconfortável se outro estudante da área da saúde soubesse mais sobre um tópico que eu" apresentou maior dispersão nas respostas.

Quanto ao fator 3 "Atenção à saúde centrada no paciente", o item 29, "Na minha profissão são necessárias habilidades de interação e cooperação com os pacientes (situação do paciente)" se sobressaiu pois, apresentou concordância de 90,3\% entre os acadêmicos de enfermagem, seguido pelos itens $26(87,1 \%), 32(87,1 \%), 34(83,8 \%), 35$ $(83,8 \%), 28(80,6 \%), 36(80,6 \%)$ que sinalizam o valor atribuído as relações entre profissionais, pacientes e familiares e como o trabalho articulado e competente dos profissionais que integram equipes de saúde favorecem a qualidade dos cuidados prestado.

A partir da categorização das respostas atribuídas a questão aberta, e análise à luz dos referenciais adotados, identificou-se que a compreensão dos acadêmicos de enfermagem sobre o que é interprofissionalidade divergiu e aproximou-se a conceitos similares e complementares, porém não intercambiáveis. As categorias que emergiram na análise dos dados qualitativos foram: colaboração interprofissional, interdisciplinar, multidisciplinar, equipe agrupamento, equipe integração e interprofissionalidade, conforme observado na Figura 1. 
Tabela 2: Distribuição das médias e desvios padrões das respostas dos acadêmicos do curso de enfermagem nos itens da Readiness for Interprofessional Learning Scale. Brasília, DF, Brasil, 2020.

Fatores/Itens RIPIS Medidas de tendência central

\section{Fator 1. Trabalho em equipe e colaboração} $M=4,63$

1

2

3

4

5

6

7

8

9

12

13

14

15

16

40

Fator 2. Identidade profissional $M=4,32$

10

11

21

38

Fator 3. Atenção à saúde centrada no paciente

\section{$M=4,69$}

25

26

27

28

29

31

32

33

34

35

36

37

39
M

DP

$\begin{array}{cc}4,7 & 0,5 \\ 4,6 & 0,9 \\ 4,8 & 0,4 \\ 4,6 & 0,8 \\ 4,6 & 0,5 \\ 4,4 & 0,6 \\ 4,6 & 0,6 \\ 4,7 & 0,5 \\ 4,6 & 0,5 \\ 2,0 & 1,3 \\ * & \\ 4,7 & 0,4 \\ 4,7 & 0,4 \\ 4,6 & 0,7 \\ 4,8 & 0,4 \\ 4,6 & 0,6\end{array}$

$1,4 \quad 0,8$

1,6

1,2

1,8

1,1

4,2

0,7

$4,5 \quad 0,6$

$4,8 \quad 0,4$

$4,4 \quad 0,8$

$4,8 \quad 0,4$

$4,9 \quad 0,3$

$4,6 \quad 0,8$

$4,7 \quad 0,6$

$4,2 \quad 0,8$

$4,8 \quad 0,3$

$4,8 \quad 0,3$

$4,7 \quad 0,5$

$4,6 \quad 0,6$

$4,7 \quad 0,4$

Legenda: $\mathrm{M}=$ Média, $\mathrm{DP}=$ Desvio padrão, $\mathrm{MD}=$ mediana, ${ }^{*}$ Média Reversa.

Fonte: Dados da Pesquisa, 2020. 


\begin{tabular}{|c|c|}
\hline Categorias & Respostas atribuídas a questão "O que é interprofissionalida" \\
\hline $\begin{array}{l}\text { Colaboração } \\
\text { interprofissional }\end{array}$ & $\begin{array}{l}\text { E02: Diferentes categorias profissionais trabalhar em equipe sem qualquer hierarquização e todos } \\
\text { compreenderem a importância desempenhada por cada profissional }\end{array}$ \\
\hline Interdisciplinar & $\begin{array}{l}\text { E01: A junção de profissionais diferenciados para atuar naquele propósito buscando o mesmo } \\
\text { resultado. } \\
\text { E05: Diversas profissões trabalhando junto em torno de um objetivo comum. } \\
\text { E26: São trabalhos que se realizam entre duas ou mais profissões ou profissionais de áreas diversas } \\
\text { com objetivo em comum. }\end{array}$ \\
\hline Multidisciplinar & $\begin{array}{l}\text { E28: Algo que percorre sob a competência de vários profissionais. } \\
\text { E31: Todas as profissões juntas em prol de um objetivo, cada uma na sua função. } \\
\text { E16: Ações e condutas tomadas por pessoas de diferentes profissões, com um objeto e objetivo único. }\end{array}$ \\
\hline Equipe agrupamento & $\begin{array}{l}\text { E19: Profissionais de áreas distintas trabalhando juntos. } \\
\text { E14: Entendo como trabalho em equipe de várias profissões juntas. }\end{array}$ \\
\hline Equipe integração & $\begin{array}{l}\text { E27: Interprofissionalidade pode ser entendido como o atendimento/trabalho conjunto dos } \\
\text { profissionais das mais diversas áreas de atenção em saúde. } \\
\text { E25: Trabalho conjunto de várias áreas da saúde em prol de uma assistência cada vez mais efetiva. } \\
\text { E22: A construção conjunta de saberes para a assistência em saúde integral e sem fragmentação de } \\
\text { conhecimentos. }\end{array}$ \\
\hline Interprofissionalidade & $\begin{array}{l}\text { E07: Interprofissionalidade é a capacidade de profissionais de diferentes áreas atuarem em conjunto } \\
\text { colaborando para o atendimento integral ao indivíduo havendo respeito a singularidade de cada um e } \\
\text { entre eles tomando decisões em grupo e trocando experiências para o crescimento tanto profissional } \\
\text { como pessoal auxiliando na prática eficaz do cuidado ao ser humano. } \\
\text { E08: A interprofissionalidade é o trabalho que abrange todas as profissões onde o foco central não } \\
\text { está em apenas uma, mas em um trabalho em equipe independente da área de formação daquele } \\
\text { determinado profissional. Na área da saúde ela é fundamental para que o paciente seja tratado como } \\
\text { um todo tendo em vista que o serviço será prestado por diversos profissionais sendo eles de } \\
\text { enfermagem nutrição medicina trabalhando em conjunto para o bem do paciente. }\end{array}$ \\
\hline
\end{tabular}

FIGURA 1: Compreensão dos acadêmicos de enfermagem quanto ao significado de interprofissionalidade. Brasília, DF, Brasil, 2020. Fonte: Dados da Pesquisa, 2020.

\section{DISCUSSÃO}

Conhecer a disponibilidade de acadêmicos para o conhecimento compartilhado possibilita a identificação de fragilidades e potencias para a adequação de currículos e implementação de estratégias que fortaleçam a abordagem da interprofissionalidade na academia. Nesta pesquisa a aplicação da RIPLS a um grupo de acadêmicos do oitavo semestre de enfermagem evidenciou resultados favoráveis a EIP para os três fatores avaliados.

A EIP prevê a criação de condições que ampliem as relações entre os membros de diferentes profissões da área da saúde. Entre seus objetivos busca-se a superação do modelo biomédico, ainda predominante no Brasil, e a entrega de melhores resultados aos usuários do sistema único de saúde, a partir da reorganização do trabalho entre profissionais e das práticas de saúde ${ }^{24}$.

A interprofissionalidade quando inserida como elemento curricular apoia a formação de profissionais com habilidades para o trabalho colaborativo, horizontal e centrado nas necessidades de indivíduos e comunidades. 0 distanciamento entre estudantes durante a graduação ressalta disputas e hostilidades que repercutem negativamente no processo de estreitamento das relações entre profissionais. Estratégias curriculares que incluem a EIP reduzem as lacunas associadas a formação e colaboram para desenvolvimento de competências colaborativas ${ }^{25}$.

Com relação ao fator 1 "trabalho em equipe e colaboração", estudo similar ${ }^{12}$ também obteve alta concordância nos itens 1, 2 e 3. Estes estão relacionados com a aprendizagem compartilhada e a consequência positiva no serviço prestado ao paciente. Mesmo ao final, o grupo pesquisado reconheceu a importância da aprendizagem compartilhada, dado este corroborado por pesquisa que identificou que alunos que estão no final do curso possuem menor potencial para produzir habilidades colaborativas ${ }^{12}$.

A importância da EIP na graduação se dá por vários aspectos, como a compreensão da prática colaborativa que se estende além do reconhecimento da atuação de outros profissionais, mas da percepção da sua própria área de atuação. A enfermagem deve considerar suas competências de modo a qualificar o cuidado e assistência ao paciente, a exemplo os diagnósticos de enfermagem ${ }^{26}$.

No fator 2 "identidade profissional" o item 21 "Eu me sentiria desconfortável se outro estudante da área da saúde soubesse mais sobre um tópico que eu" apresentou maior dispersão, assim como em estudo de $2017^{12}$, em que os acadêmicos de enfermagem participantes também apresentaram grande discordância para esse item. A enfermagem é 
essencial para o funcionamento adequado dos serviços de saúde no Brasil e no mundo, sendo necessário a inovação e expansão das ações desenvolvidas pela profissão por meio das práticas avançadas de enfermagem ${ }^{27}$. Sabe-se que apesar disso a profissão enfrenta desafios e é marcada por estigmas que são reforçados inclusive por meios de comunicação ${ }^{28}$. Esse desconforto apresentado pelo item 21 é consequência das experiências vividas e relatadas de profissionais da enfermagem com relação a outras profissões no ambiente de trabalho, surgindo a necessidade de preservar sua autonomia e evitar situações desconfortáveis ${ }^{28}$.

Em relação ao fator 3 "atenção à saúde centrada no paciente", um estudo mais amplo que inclui diferentes cursos da área da saúde ${ }^{12}$ demonstrou que os estudantes de enfermagem que estão nos anos iniciais da formação apresentam maior disponibilidade para desenvolvimento da atenção centrada no paciente. Já o presente estudo demonstrou que os acadêmicos no último período apresentam alta disponibilidade para esse fator. 0 item 26 "estabelecer uma relação de confiança com meus pacientes é importante para mim (situação do paciente)", se destacou em ambos os estudos, neste houve grande concordância $(87,10 \%)$ e no estudo mais amplo houve uma pequena diminuição ao longo dos anos na formação.

Em relação aos dados qualitativos os conhecimentos dos estudantes sobre a definição da interprofissionalidade ${ }^{1}$, se aproximam de conceitos diferentes como colaboração interprofissional ${ }^{21}$, interdisciplinaridade ${ }^{21}$, multidisciplinaridade ${ }^{21}$, trabalho em equipe agrupamento e equipe integração ${ }^{22}$, o que denota uma reprodução do que já foi vivenciado durante a graduação. O projeto pedagógico do curso (PPC) de enfermagem da instituição estudada, demonstra que os objetivos propostos para a graduação incluem o desenvolvimento de habilidades em equipe interdisciplinar e multidisciplinar, não sendo incluso a EIP e a interprofissionalidade ${ }^{29}$.

Os projetos de extensão são grandes oportunidades para o desenvolvimento das habilidades do trabalho interprofissional e colaborativo em saúde ${ }^{30,31}$. Embora seja enfatizado no PPC do presente estudo a necessidade de atividades complementares como a extensão, entre os projetos executados a âmbito do curso de enfermagem estudado somente alguns integram cursos de diferentes profissões ${ }^{32}$.

No contexto estudado identifica-se a necessidade de ampliação da abordagem da EIP, para que os acadêmicos desenvolvam competências colaborativas. Uma alternativa é a implementação de estratégias de ensino aprendizagem e metodologias ativas como: Aprendizado baseado em seminário, Aprendizado baseado em observação (shadowing), Aprendizado baseado em problemas, Aprendizado baseado em simulação, Aprendizado baseado na prática clínica, Aprendizado E-learning (ex: discussões online) e Aprendizado misturado (integrando e-learning com outro método tradicional) ${ }^{33}$.

Os docentes dos cursos da saúde têm papel fundamental na introdução e manutenção da EIP na graduação desses acadêmicos. Os estudantes de enfermagem e medicina que participaram de um estudo de $2018^{25}$ demonstraram que os professores são importantes mediadores para que ocorra o aprendizado compartilhado, sendo assim é relevante um corpo docente composto por diversas áreas, que permita essa integração. Desde a formação docente é necessário que a EIP seja introduzida, para que ele tenha maiores experiências com o ensino-aprendizagem pautado na EIP e consequentemente haja um comprometimento com essa prática pedagógica, já que sua atuação contribuirá para que os estereótipos acerca das profissões da saúde sejam rompidos ${ }^{25}$.

A atuação profissional tem forte relação com a formação acadêmica dos estudantes, sendo necessário que estes tenham como um dos aspectos do processo educativo a interprofissionalidade, que deve estar presente nas práticas em saúde desenvolvidas durante o processo de formação ${ }^{34}$. A reflexão constante dos modelos de ensino dos estudantes da saúde é uma importante estratégia para o fortalecimento do SUS, sendo assim, a EIP deve ser adaptada para o contexto brasileiro e para nosso sistema único de saúde, para que suas competências sejam exploradas e as iniciativas pautadas na EIP sejam implementadas e alcancem maior visibilidade ${ }^{35}$.

\section{Limitações do estudo}

Como limitações citamos o número de participantes da pesquisa e a necessidade de realização da abordagem dos potenciais participantes por meio virtual, devido a situação da pandemia instaurada no país.

\section{CONCLUSÃO}

Os acadêmicos de enfermagem participantes da pesquisa apresentaram disponibilidade para a EIP nos três fatores avaliados pela escala RIPLS, demonstrando disposição para o trabalho colaborativo e para a interprofissionalidade.

Com relação a compreensão do que é interprofissionalidade, os resultados evidenciaram que ainda há lacunas nas interpretações deste termo, a qual se confunde com termos similares. 
O presente estudo oferece um diagnóstico situacional para o curso de graduação pesquisado e pode agregar na ampliação da discussão acerca da temática e da produção de estratégias para o fortalecimento da EIP no meio acadêmico.

\section{REFERÊNCIAS}

1. Barr H, Freeth D, Hammick M, Koppel I, Reeves S. Effective interprofessional education: arguments, assumption \& evidence. Oxford: Blackwell; 2005.

2. Ceccim, RB. Connections and boundaries of interprofessionality: form and formation. Rev. Interface (Botucatu) [Internet]. 2018 [cited 2021 May 18]; 22(1):1739-49. DOI: https://doi.org/10.1590/1807-57622018.0477.

3. Peduzzi M, Oliveira MAC, Silva JAM, Agreli HLF, Neto MVM. Trabalho em equipe, prática e educação interprofissional. In: Clínica médica: atuação da clínica médica, sinais e sintomas de natureza sistêmica, medicina preventiva, saúde da mulher, envelhecimento e geriatria. Barueri: Manole; 2016 [cited 2020 Jan 04]. p. 1-9. Available from: https://edisciplinas.usp.br/pluginfile.php/3011330/mod_resource/content/1/Trabalho\%20em\%20equipe.pdf.

4. Conselho Nacional de Educação (Br). Resolução no 3 de 7 de novembro de 2001. Institui Diretrizes Curriculares Nacionais do Curso de Graduação em Enfermagem. Brasília (DF): CNE/CES; 2001 [cited 2020 Apr 13]. Available from: http://portal.mec.gov.br/cne/arquivos/pdf/CES03.pdf

5. Conselho Nacional de Saúde (Br). Resolução № 569, de 8 de dezembro de 2017. Princípios Gerais para as Diretrizes Curriculares Nacionais dos Cursos de Graduação da Área da Saúde. Brasília (DF): CNS; 2017 [cited 2020 Jan 05]. Available from: https://conselho.saude.gov.br/resolucoes/2017/Reso569.pdf.

6. Organização Mundial da Saúde (OMS). Framework for action on interprofessional education and collaborative practice [internet]. Geneva: OMS; 2010. [cited 2020 Jan 4]. Available from: http://www.fnepas.org.br/oms traduzido 2010.pdf.

7. Freitas MAO, Demarchi GSS, Rossit RAS. Interprofessional Education in Master's and Doctoral programs: postgraduate students' perception. Interface (Botucatu) [Internet]. 2018 [cited 2021 May 18]; 22(2):1647-59. DOI: https://doi.org/10.1590/180757622017.0644

8. Costa MV. The interprofessional education in Brazilian context: some reflections. Interface (Botucatu) [Internet]. 2016 [cited 2021 May 18]; 20(56):197-8. DOI: https://doi.org/10.1590/1807-57622015.0311.

9. Peduzzi M, Normam I, Coster S, Meireles E. Adaptación transcultural y validación de la Readiness for Interprofessional Learning Scale en Brazil. Rev Esc Enferm USP [Internet]. 2015 [cited 2020 Jan 20]; 49(2):7-15. DOI: http://dx.doi.org/10.1590/S0080623420150000800002.

10. Rodrigues JRS, Soares FJP, Alcântara RC. Perspectivas para a educação interprofissional em um hospital de trauma. Rev. CIAIQ. [Internet]. 2018 [cited 2020 Jan 28]; 2:62-71. Available from: https://proceedings.ciaiq.org/index.php/ciaiq2018/article/view/1765/1718

11. Souto TS, Batista SH, Batista NA. The interprofessional education in educational Psychology: student's perspectives. Psicologia: ciência e profissão [Internet]. 2014 [cited 2020 Mar 02]; 34(1):32-45. DOI: https://doi.org/10.1590/S1414-98932014000100004.

12. Nuto SAS, Júnior FCML, Camara AMCS, Gonçalves CBC. An Evaluation of Health Sciences Students' Readiness for Interprofessional Learning. Rev. bras. educ. med [Internet]. 2017 [cited 2020 Mar 12]; 41(1):50-7. DOI: http://dx.doi.org/10.1590/1981-52712015v41n1rb20160018.

13. Câmara AMCS. Educação interprofissional no PET-saúde: Cenário para o desenvolvimento de Práticas e Competências Colaborativas na área da saúde [Tese de doutorado]. Brasília: Universidade de Brasília, Faculdade de Ceilândia, Programa de Pós-Graduação em Ciências e Tecnologia em Saúde. 2015 [cited 2020 Mar 10]. 176 p. DOI: http://dx.doi.org/10.26512/2015.07.T.18717.

14. Vilela RQB, Amado E. Interprofessional education and collaborative practice in intensive care: the health professional perspective. Braz Ap Sci Rev [Internet]. 2018 [cited 2020 Jan 28]; 2(4):1247-68. Available from: https://www.brazilianjournals.com/index.php/BASR/article/view/510.

15. Barbosa GR. Readiness of students from eight health undergraduation courses at a brazilian public university for interprofessional education [Tese de doutorado]. Campinas: Repositório da produção científica e intelectual da Unicamp on line. 2020 [cited 2020 Jan 20]. 88 p. Available from: http://repositorio.unicamp.br/jspui/handle/REPOSIP/346070.

16. Candido LO, Rossit RAS, Oliveira RC. Professional insertion of the graduates from a physical education course with emphasis on health training. Rev Trab Educ e Saúde [Internet]. 2018 [cited 2020 Jun 20]; 16(1):305-18. DOI: https://doi.org/10.1590/19817746-sol00096.

17. Uchôa PA. Estágio integrado em Saúde e aprendizagem interprofissional: percepção dos discentes [Dissertação]. Faculdade de Medicina, Programa de Pós Graduação em ensino na saúde, Universidade Federal de Alagoas. 2018 [cited 2020 Oct 10]. 100 p. Available from: http://www.repositorio.ufal.br/handle/riufal/3409.

18. Tompsen NN, Meireles E, Peduzzi M, Toassi RFC. Interprofessional education in undergraduation in dentistry: curricular experiences and student availability. Rev Odontol UNESP [Internet]. 2018 [cited 2020 May 5]; 47(5):309-20. DOI: https://doi.org/10.1590/1807-2577.08518.

19. Souza A. Formação Profissional em Saúde na Perspectiva do Trabalho em Equipe e da Integralidade no Cuidado: Percepção do Estudante [Dissertação]. São Paulo: Universidade Federal de São Paulo, Campus São Paulo. 2014 [cited 2020 May 18]. 97 p. Available from: http://www.repositorio.unifesp.br/jspui/handle/11600/41920.

20. Oliveira DC. Análise de conteúdo temático-categorial: uma proposta de sistematização. Rev. Enferm. UERJ [internet]. 2008 [cited 2020 Oct 20]; 16(4):569-76. Available from: http://files.bvs.br/upload/S/0104-3552/2008/v16n4/a569-576.pdf. 
21. Khalili H, Thistlethwaite J, El-Awaisi A, Pfeifl A, Gilbert J, Lising D, et al. Orientação para a educação interprofissional global e pesquisa sobre a prática colaborativa: Documento de trabalho. Publicação conjunta do Interprofessional Research.Global e da Interprofessional.Global [Internet]. 2019 [cited 2020 Oct 10]. Available from: https://interprofessional.global/wpcontent/uploads/2019/10/Orienta\%C3\%A7\%C3\%A3o-sobre-pesquisa-global-em-educa\%C3\%A7\%C3\%A3o-interprofissional-epr\%C3\%A1tica-colaborativa-Documento-de-trabalho_FINAL-WEB.pdf.

22. Peduzzi M. Equipe Multiprofissional de Saúde: conceito e tipologia. Rev saúde Públ [Internet]. 2001 [cited 2020 oct 10]; 35(1):103-9. DOI: https://doi.org/10.1590/S0034-89102001000100016.

23. Conselho Nacional de Saúde (Br). Resolução no 466 de 12 de dezembro de 2012. Diretrizes e normas regulamentadoras de pesquisas envolvendo seres humanos. Brasília (DF): CNS; 2012. [cited 2020 Oct 10]. Available from: http://bit.ly/1mTMIS3.

24. Griggio AP, Silva JAM, Rossit RAS, Mieiro DB, Miranda FM, Mininel VA. Analysis of an interprofessional education activity in the occupational health field. Rev Latino-Am Enferm [Internet]. 2020 [cited 2021 Mar 05]; 28:e3247. DOI: http://dx.doi.org/10.1590/1518-8345.3228.3247.

25. Santos LC, Simonetti JP, Cyrino AP. Interprofessional education in the undergraduate Medicine and Nursing courses in primary health care practice: the students' perspective. Rev. Interface-Comunic, Saúde, Educ [Internet]. 2018 [cited 2021 Mar 12]; 22(2):1601-11. DOI: https://doi.org/10.1590/1807-57622017.0507.

26. Santos GLA, Valadares GV, Santos SS, Moraes CRBM, Mello JCM, Vidal LLS. Interprofessional collaborative practice and nursing care. Esc Anna Nery [Internet]. 2020 [cited 2021 Mar 20]; 24(3):1-6. DOI: https://doi.org/10.1590/2177-9465-ean-2019-0277.

27. Honig J, Doyle-Lindrud S, Dohrn J. Moving towards universal health coverage: advanced practice nurse competencies. Rev. Latino-Am. Enferm. [Internet]. 2019 [cited 2021 Mar 10]; 27:e3132. DOI: https://doi.org/10.1590/1518-8345.2901.3132.

28. Silva JC, Moraes H, Araújo F, Araújo MK, Oliveira MR. Where is nursing? The (in)visibility of this professional category in the media. Rev. Enferm foco [Internet]. 2020 [cited 2021 Mar 22]; 11(2):48-54. DOI: https://doi.org/10.21675/2357707X.2020.v11.n2.2968.

29. Departamento de Enfermagem (UnB). Comissão de atualização: Membros do Núcleo Docente Estruturante. Projeto pedagógico do curso de graduação em enfermagem [Internet]. Brasília (DF): Departamento de Enfermagem; 2017. Available from: PPC_2017_atualizado_Enfermagem.pdf (unb.br).

30. Salomão AFS, Cunha ACM, Silva JCN, Correia HPC, Yogui JOS, Camargos CCRL et al. Interprofessional education in the context of Primary Health Care: experience report. Rev. de APS [Internet]. 2018 [cited 2021 Mar 02]; 21(4):747-56. DOI: https://doi.org/10.34019/1809-8363.2018.v21.16305.

31. Costa MV, Azevedo GD, Vilar MJP. Aspectos institucionais para a adoção da Educação Interprofissional na formação em enfermagem e medicina]. Rev Saúde Debate [Internet]. 2019 [cited 2021 Apr 25]; 43(1):64-76. DOI: https://doi.org/10.1590/0103-11042019S105.

32. Universidade de Brasília- Faculdade de Ciências da Saúde [internet]. Enfermagem: Projetos de Extensão de Ação Contínua (PEAC) [cited 2021 Apr 24]. Available from: http://fs.unb.br/projetos-de-extensao-graduacao-enfermagem.

33. Reeves $\mathrm{S}$. Why we need interprofessional education to improve the delivery of safe and effective care. Rev. Interface (Botucatu) [Internet]. 2016 [cited 2021 Mar 30]; 20(56):185-97. DOI: https://doi.org/10.1590/1807-57622014.0092.

34. Conselho Nacional de Saúde (Br). Resolução № 573, de 31 de janeiro de 2018. Recomendações do Conselho Nacional de Saúde à proposta de Diretrizes Curriculares Nacionais (DCN) do curso de graduação Bacharelado em Enfermagem [internet]. Brasília (DF): CNS; 2018 [cited 2020 Apr 10]. Available from: https://conselho.saude.gov.br/resolucoes/2018/Reso573.pdf.

35. Costa MV. A potência da educação interprofissional para o desenvolvimento de competências colaborativas no trabalho em saúde. In: Toassi FC, organizadora. Interprofissionalidade e formação na saúde: onde estamos?. Porto Alegre: Rede Unida; 2017. [cited 2021 Apr 02] p. 15. Available from:

https://www.lume.ufrgs.br/bitstream/handle/10183/183942/001064798.pdf?sequence=1\&isallowed. 